\title{
AN EXPERIMENTAL STUDY OF THE LARGE DEFORMATION OF PLASTIC HINGES
}

\author{
I. K. McIvor, W. J. Anderson and M. BiJaK-Zochowski† \\ The University of Michigan, Ann Arbor, MI 48109, U.S.A.
}

(Received 29 March 1976; revised 26 July 1976)

\begin{abstract}
Recent applications of structural plasticity to areas such as vehicle crashworthiness has led to interest in the large deformation plastic collapse of general frames. Even when displacements are comparable to the original structural dimensions, the plasticity is confined to localized regions or "hinges". This paper reports an experimental study of the behavior of such hinges in thin walled structural members. Due to local deformation the load carrying capacity of the hinge significantly decreases at large rotations. In a companion paper [4] a structural constitutive theory is proposed to account for this behavior. Numerical data for this theory is obtained in the present paper. Finally test results are given for a large deformation combined loading test designed to validate the theory of [4]. The experimental results are in good agreement with the theoretical predictions.
\end{abstract}

\section{INTRODUCTION}

The concept of a "plastic hinge" plays a central role in the limit analysis of structures. The physical basis for this idealization is well established. Discussion and references to the original literature may be found in a number of standard textbooks [1-3]. In the classical formulation the hinge transmits the applied moment without rotation until the yield moment is reached. At the yield moment it permits arbitrary rotation. The collapse load is associated with a distribution of hinges that permits infinitesimal rigid body motion of all or part of the structure.

In recent applications of structural plasticity to areas such as vehicle crashworthiness, the collapse load is of little direct interest. It is the behavior of the structure during collapse which is significant. Nevertheless, the concept of a plastic hinge remains a useful idealization. Even at large deformations the plasticity is confirmed to localized regions. A cantilever beam subjected to bending and torsion is shown in Fig. 11. The characteristic feature of the deformation is clearly a "plastic hinge" permitting finite rotations. Within the hinge region itself, substantial local deformation has occurred.

In a companion paper [4] a structural plasticity theory for the large deformation of general frames is derived on the assumption that the plasticity is confined to idealized hinges. In the large deformation range, however, the load carrying capacity of the hinge decreases significantly due to local deformation. Local deformation cannot, of course, be directly computed in the context of a structural theory. On the structural scale its effect is an inherent part of the constitutive behavior of the hinge. The formulation in [4] incorporates such a constitutive theory.

In this paper the results of an experimental study on the large deformation behavior of plastic hinges are presented. The study is intended to demonstrate the characteristic features of this behavior as well as to provide specific data for the analysis in [4]. Finally, to validate the predictive capability of the theory, a large deformation combined loading test was developed. The hinge is subjected to nonproportional bending, torsion, and axial loads with finite rotations exceeding $45^{\circ}$. The predicted force-deformation curve is in good agreement with the test results.

\section{EXPERIMENTAL HINGE TESTS}

An experimental program was designed to study the behavior of plastic hinges subjected to large rotations. In addition to demonstrating characteristic features, the program obtained data for the constitutive theory proposed in [4]. We briefly summarize the pertinent equations. Detailed discussion is given in [4].

We assume the behavior of the hinge is determined by a scaler generalized yield function

$$
f(\xi)=1
$$

†Visiting Research Associate, on Leave from Technical University of Warsaw, Poland. 
where

$$
\xi_{\mathrm{j}}=Y_{j} / \alpha_{i} \quad j=1,2,3,4
$$

in which the four elements of $\mathbf{Y}$ denote the current values of the axial force, bending moments about the principal axes, and torque respectively. (For convenience the dependence of $f$ on transverse shear is neglected). Thus, the components of $\boldsymbol{\xi}$ are normalized stress resultants in local beam coordinates. The scaling parameters $\alpha_{j}$ are considered as constitutive properties of the hinge whose value depends upon the history of plastic deformation. In particular we assume

$$
\alpha_{1}=\alpha_{i}\left(\theta_{i}\right) \quad j=1,2,3,4
$$

where the scalers $\theta_{i}$ denote the accumulated plastic deformation in extension, biaxial bending, and torsion respectively. Precise definitions of $\theta_{j}$ are given in [4].

The experimental task is thus to determine the parameters $\alpha_{j}$. With the assumption embodied in (3), it is sufficient to conduct tests in extension, pure bending, and torsion. A number of factors must be considered in the design of the experiments. These include (a) The beain cross section must be supported at points of loading and reaction to prevent local crushing due to extraneous stress concentrations. (b) The test must be displacement controlled so that the specimen does not collapse in the "softening" region of the load-displacement curve. (c) The loading fixture must apply a constant direction load over the entire large deformation range. (d) Specimens must be designed to insure a gage length which is characteristic of hinge formation. Preliminary tests in unconstrained specimens indicated that twice the beam thickness is an appropriate characteristic length.

The last item merits further discussion. In principal the stress state in the specimen is homogenous. Preliminary tests on unconstrained specimens showed, however, that a region of localized deformation always formed. Even at large rotations the length of this local deformation was less than twice the beam thickness. Thus this distance was taken as the characteristic length associated with hinge formation.

In the unconstrained specimens partial yielding occurred over the entire unsupported length prior to hinge formation. Once the hinge forms, however, the load carrying capacity decreases and the specimen outside the local deformation region unloads elastically. To control the hinge location and eliminate this overall yielding from the data, internal "plugs" were used to constrain the cross section except for the gage length of twice the beam thickness.

All tests were done using displacement controlled, static test machines. The torsion and tensile specimens required no special treatment or unusual fixtures. The experimental problem of maintaining a pure bending moment on a specimen throughout a large deformation test is more difficult. The loading device designed to accomplish this is shown in Fig. 1. The specimen is mounted between two rigid end blocks which are attached to a four point support fixture creating a pure couple about an axis in the specimen. Care must be taken to insure the hinge forms in the center of the specimen to maintain the necessary symmetry.

All specimens were 1 in square tubing with 0.075 in walls. The material was low carbon, automotive grade steel, 1018. Overall specimen lengths varied in each test, but in every case internal "plugs" were used to support the cross section at points of loading. As discussed above these plugs extended to the hinge site, leaving only a 2 in gage length free to deform. For the bending test the plugs were contoured at the hinge location to provide a gradual transition from full to no wall support. Without this precaution the hinge is likely to form near the plug end. The torsion and tension specimens employed a rounded end insert more appropriate to a hinge with an axis of symmetry along the longitudinal axis.

\section{TEST RESULTS}

The tensile test of the tube is shown in Fig. 2 in the form of a stress-strain diagram. Strain is measured over a 2 in gage length in the unsupported wall region of the test specimen. Although the test is essentially a uniaxial stress-strain test, the choice of gage length permits the results to be interpreted as a hinge test for the axial stress resultant. The results can be modeled rather well as elastic-perfectly plastic with a yield stress of 61,500 psi. Although for small strains (below 


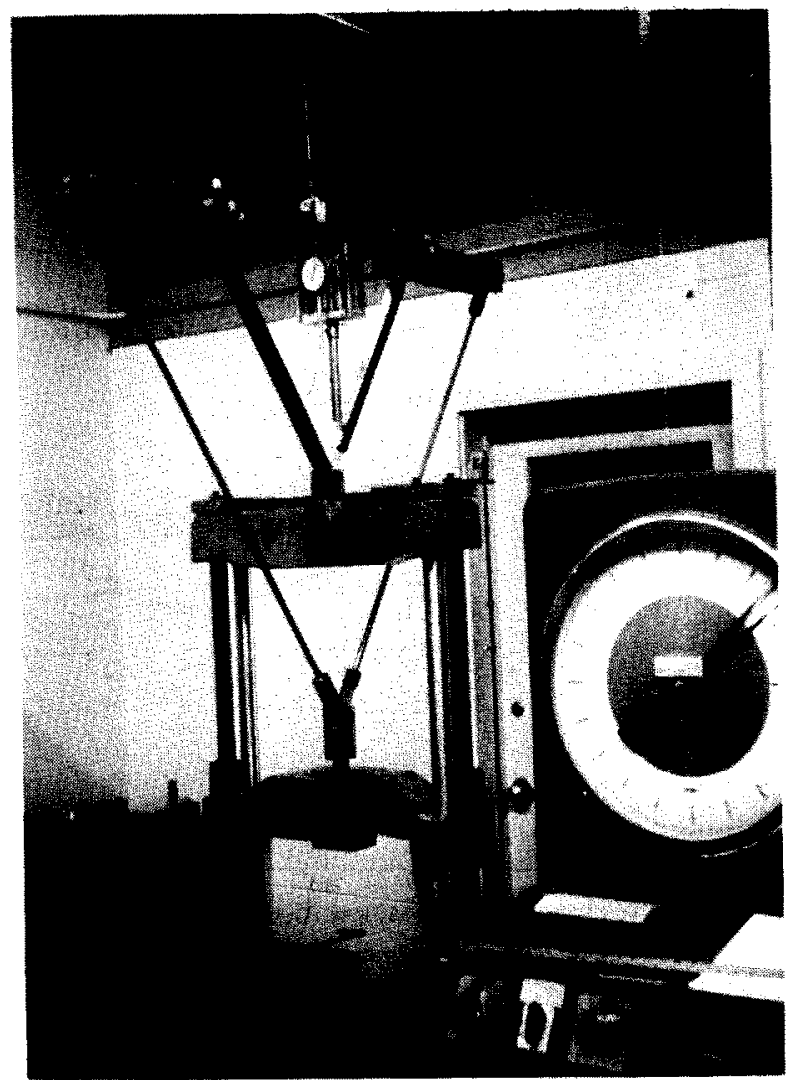

Fig. 1. Four point fixture for pure bending.

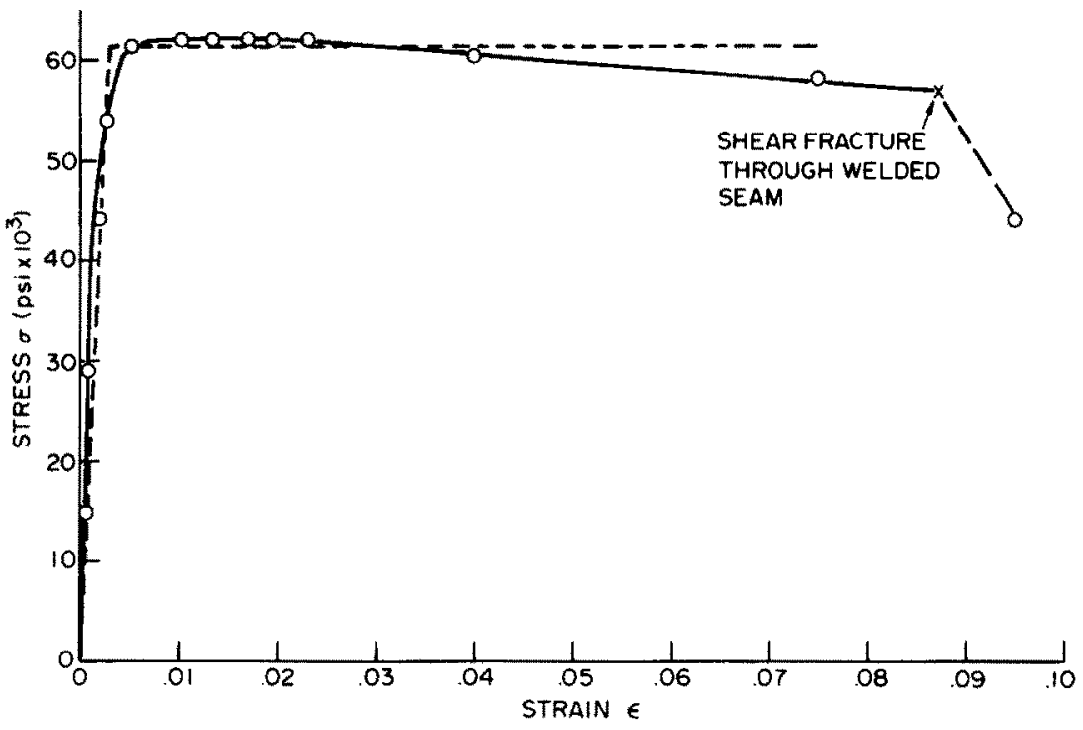

Fig. 2. Tensile test for 1 in $\times 1$ in $\times 0.075$ in tubing.

$5 \times 10^{-4}$ ), the slope of the elastic curve is nearly $30 \times 10^{6} \mathrm{psi}$, a better fit of the data over the assumed linear range is a Young's Modulus of $20 \times 10^{6} \mathrm{psi}$. The elastic-perfectly plastic approximation is shown by dashed lines in the Figure. At large strains (above 0.04 ) a waviness in the wall surface occurred. The tube failed by a shear fracture through the welded seam.

Results for the pure bending test are given in Fig. 3. The welded seam was located on the compressive side. Elastic action of the support and specimen has been numerically removed from the data. Thus the abscissa denotes plastic hinge rotation over the gage length. A stress relaxation phenomenon was observed at constant displacement. Separate curves are given for 


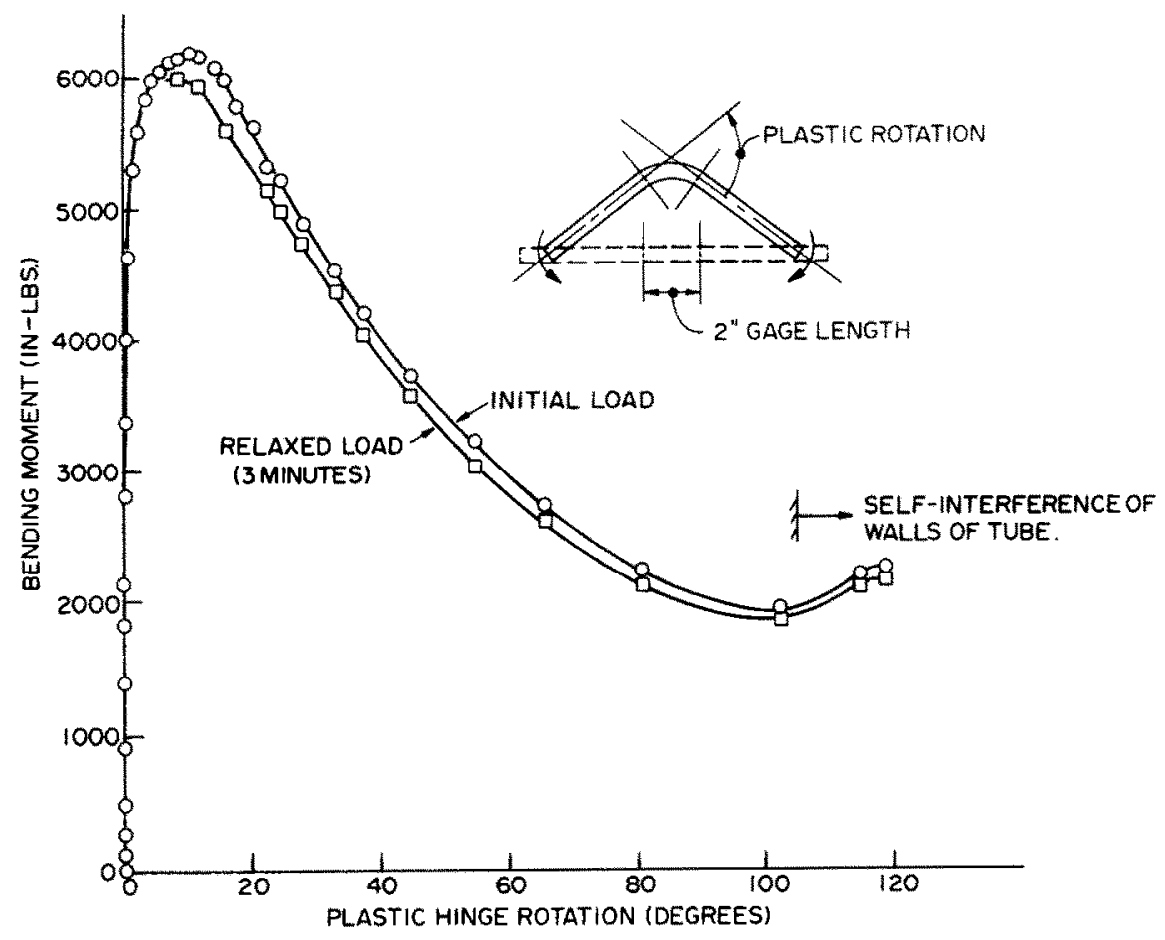

Fig. 3. Plastic hinge rotation-pure bending.

"instantaneous" bending moment at time of incremental loading and bending moment after 3 min relaxation. The latter curve can be taken as a close approximation to static values.

In contrast to the axial test, the reduction in the moment carrying capacity of the hinge with increasing rotation is dramatic. The associated development of the local deformation during hinge formation is shown in Fig. 4. This curve may be of some value for future detailed analysis of the hinge site.

Results for a pure torsion hinge are shown in Fig. 5. Again, the elastic rotation has been

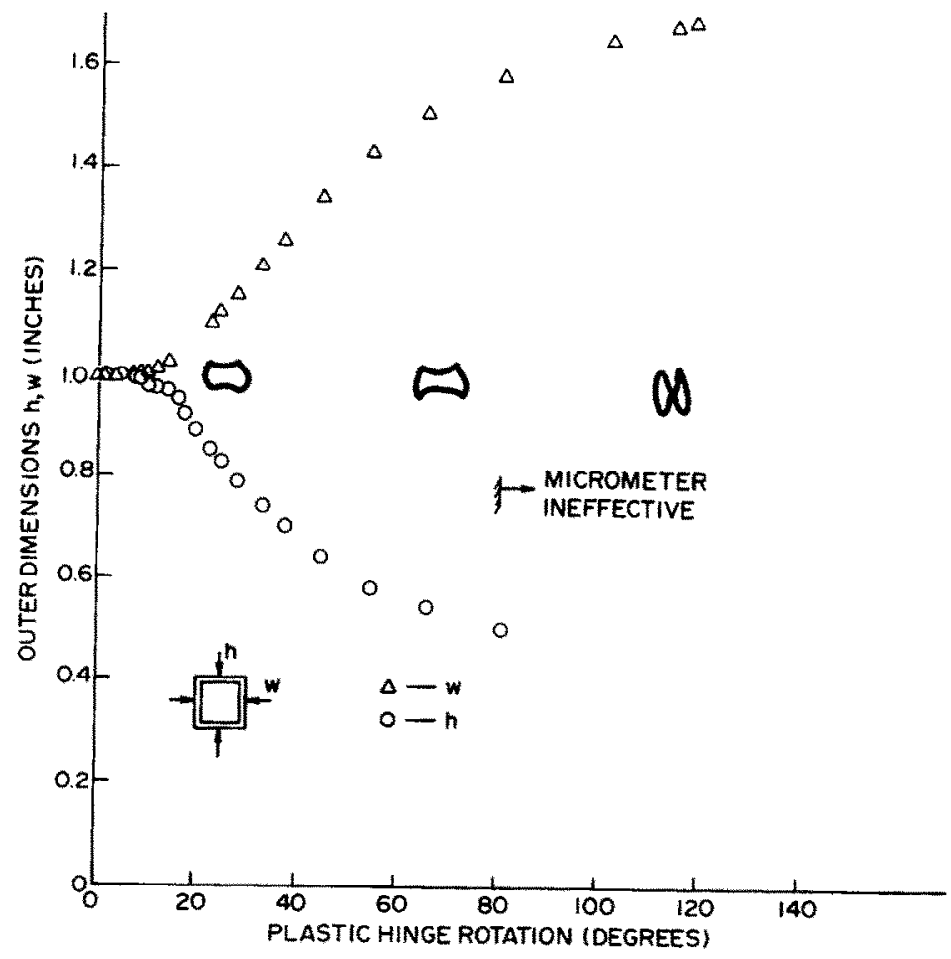

Fig. 4. Hinge deformation-pure bending. 


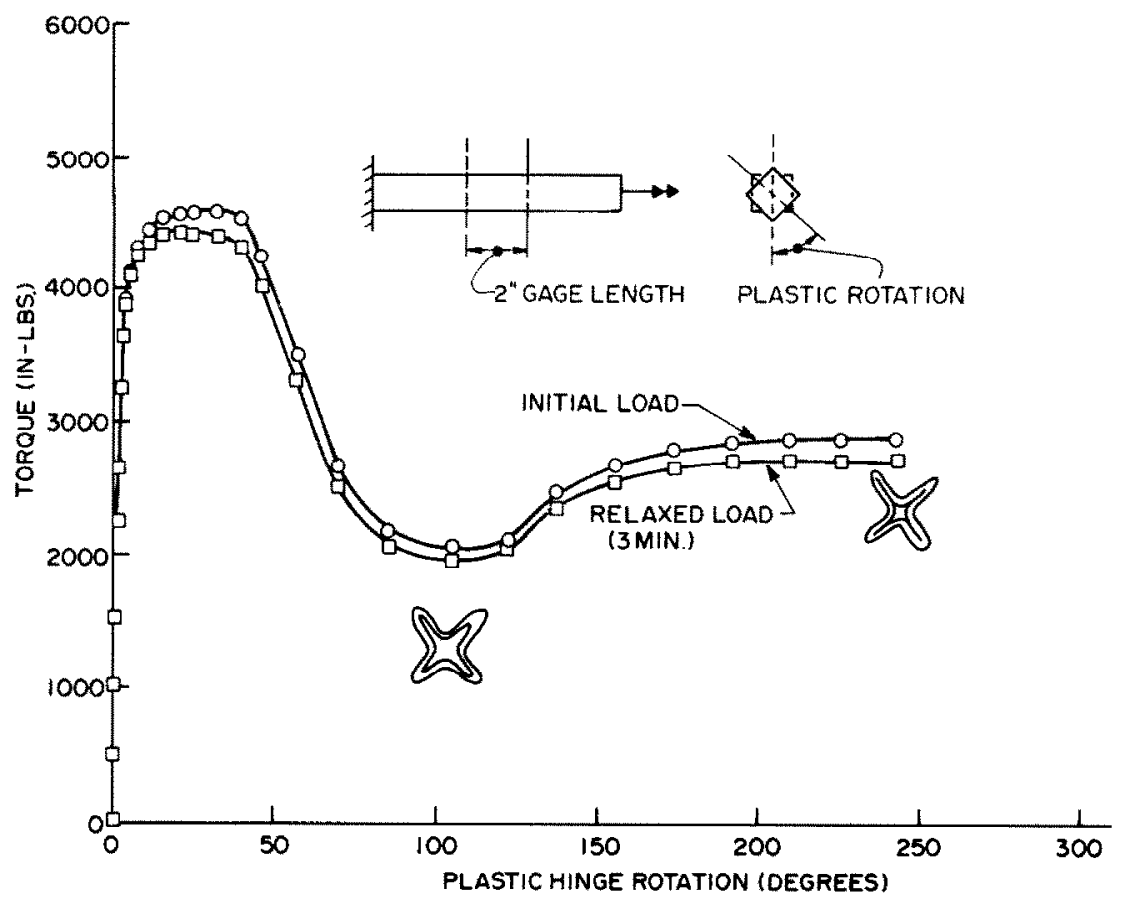

Fig. 5. Plastic hinge rotation-torsion.

removed from the data so that only plastic rotation over the 2 in gage length is given. The hinge in this case is a spiral pattern with pronounced folding of the walls into the star pattern shown. As in the bending test a load relaxation is observed.

\section{HINGE CONSTITUTIVE PARAMETERS}

To use the above data to determine the constitutive parameters $\alpha_{i}$, it is necessary to specify the yield function $f$. A reasonable choice for the functional form would be the initial yield function based on elementary beam theory and an appropriate yield stress criterion. In general this yield surface may be represented or approximated by a quadratic form. Thus for convenience in the present study we choose $f$ as the hyper-ellipse

$$
f=\sum_{j=1}^{4} \xi_{j}^{2}=1
$$

With (4) and (3) it follows that the dependence of $\alpha_{i}$ on the plastic deformation measures are given directly by the above test results.

It remains to fit the data in a form convenient for computational purposes. The characteristic shape observed in the bending and torsion tests can be approximated by an exponential function of the form

$$
\alpha=M\left\{a_{i}+b_{i}\left[1+k_{i}\left(\theta-\theta_{m}\right)\right] \exp \left[-k_{i}\left(\theta-\theta_{m}\right)\right]\right\}
$$

where $\theta$ is the accumulated plastic rotationt and $\theta_{m}$ is the value of $\theta$ corresponding to the maximum value of $\alpha$. For $\theta<\theta_{m}, i=1$ and

$$
\begin{gathered}
a_{1}=(1-f y) /(1-y) \\
b_{\mathrm{t}}=(f-1) /(1-y) . \\
y=\left(1-k_{1} \theta_{m}\right) \exp \left(k_{1} \theta_{m}\right) .
\end{gathered}
$$
in [4].

It should be noted that the measured rotation over the gage length is twice the accumulated plastic rotation as defined 
For $\theta>\theta_{m}, i=2$ and

$$
a_{2}=\beta, \quad b_{2}=f-\beta
$$

Equation (5) is a function of 6 parameters which may be used to fit the test results. Four of the parameters have simple physical interpretations and may essentially be determined by inspection; they are: $M=$ maximum elastic moment, $f=$ ratio of maximum value of $\alpha$ to $M$, $\beta$ = ratio of the asymptotic value of $\alpha$ for large $\theta$ to $M, \theta_{m}=$ value of $\theta$ at maximum value of $\alpha$.

The remaining two parameters may be considered as hardening and softening rates for the cross section. They may be obtained by minimizing the error between (6) and the test results in the two ranges $\theta<\theta_{m}, \theta>\theta_{m}$.

As shown in Fig. 2 the plastic behavior in axial extension may be modeled as perfectly plastic with a yield stress $\sigma_{y}$ of 61,500 psi. Thus the parameter $\alpha_{1}$ is a constant which is obtained from (5) by setting

$$
M=A \sigma_{y}, \quad \theta_{m}=0, \quad f=\beta=1
$$

where $A$ is the area of the cross section.

The specific numerical values obtained for the tubular beam specimen tested here are summarized in Table 1. The data shown is for a fit to the static (relaxed) curves in Figs. 3 and 5. Comparison between the test results and computed values using eqn (5) is shown in Fig. 6 for bending and Fig. 7 for torsion.

Table 1. Hinge parameters for square tubing

\begin{tabular}{cccc}
\hline $\begin{array}{c}\text { Hinge } \\
\text { parameters }\end{array}$ & $\begin{array}{c}\text { Extension } \\
\alpha_{1}\end{array}$ & $\begin{array}{c}\text { Bending } \\
\alpha_{2}, \alpha_{3}\end{array}$ & $\begin{array}{c}\text { Torsion } \\
\alpha_{4}\end{array}$ \\
\hline$M$ & $17,000 \mathrm{lbs}$. & $4,500 \mathrm{in}$ lbs. & $3,500 \mathrm{in}$ lbs. \\
$f$ & 1 & 1.34 & 1.27 \\
$\beta$ & 1 & 0.40 & 0.54 \\
$\theta_{m}$ & 0 & 0.073 & 0.244 \\
$k_{2}$ & - & 31.9 & 43.1 \\
$k_{2}$ & - & 6.20 & 7.13 \\
\hline
\end{tabular}

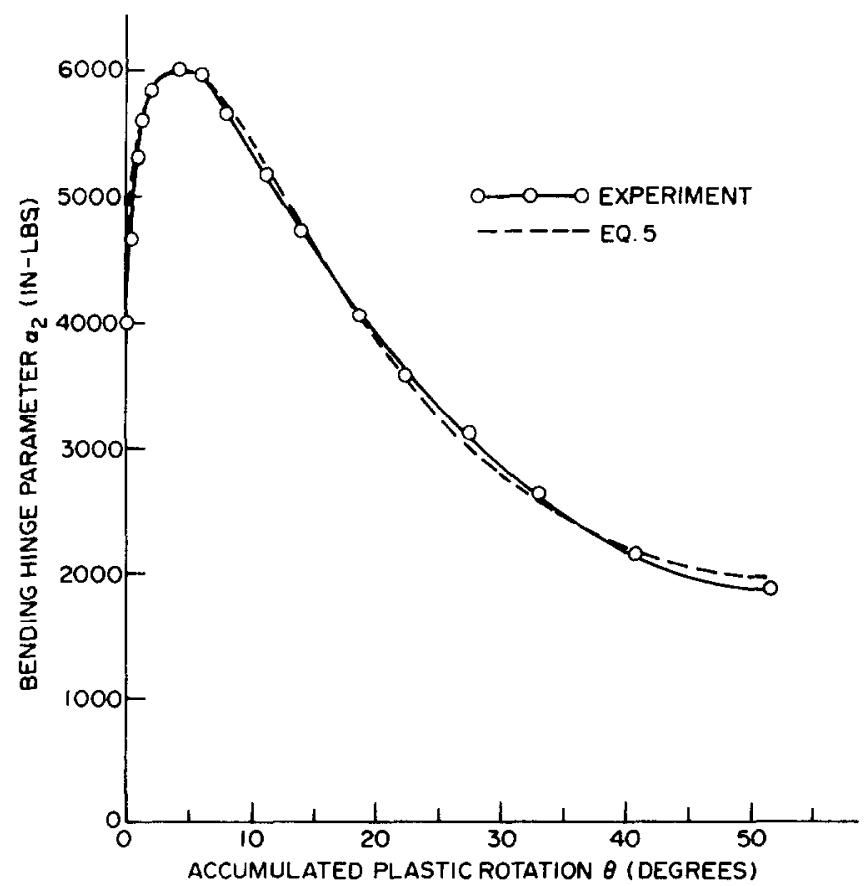

Fig. 6. Computed and experimental plastic hinge rotation in bending. 


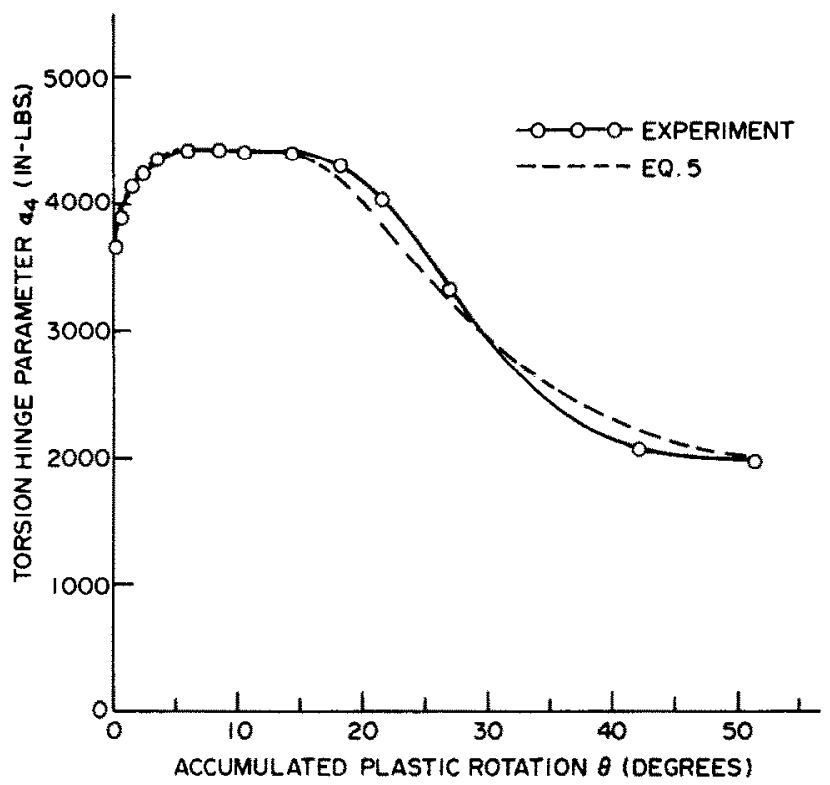

Fig. 7. Computed and experimental plastic hinge rotation in torsion.

COMBINED LOADING VALIDATION EXPERIMENT

The results of the above section provide the necessary data for the structural constitutive theory proposed in [4]. Given this data, the physical validity of the theory rests on its ability to predict hinge behavior under arbitrary loading paths. To test this predictive capability, a non-proportional combined loading experiment was devised. The test configuration is shown in Fig. 8. It consists of a double cantilever specimen, each specimen 5.5 in in length. The ends of the

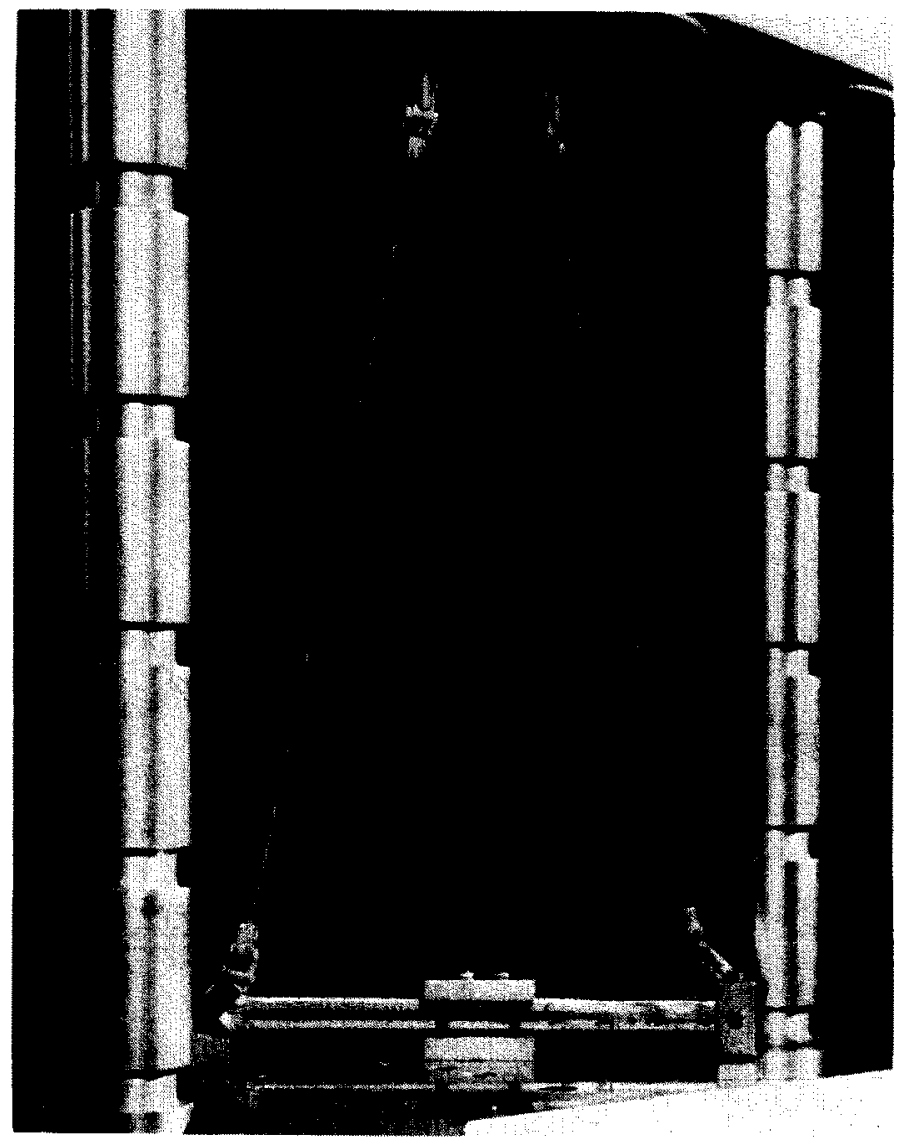

Fig. 8. Combined loading experiment. 
cantilevers are attached to rigid bars at right angles. The system is loaded through high strength cables and a system of universal joints that approximate a ball and socket joint. Symmetry insures a zero slope at the root of the cantilever. The initial geometry is shown in Fig. 9.

The cable used in loading was standard 1/8 in diameter stranded cable commonly used in aircraft control systems with breaking strength of $2000 \mathrm{lbs}$. The cable was loaded and unloaded seven times in order to align the fibers in the most inextensible position. After five cycles the load displacement curve was repeatable with a stiffness of 5,830 \#/in.

Test results are shown in Fig. 10 as the vertical force component in the cable versus the vertical displacement of the upper end of the cable. At low loads the system is elastic. A hinge

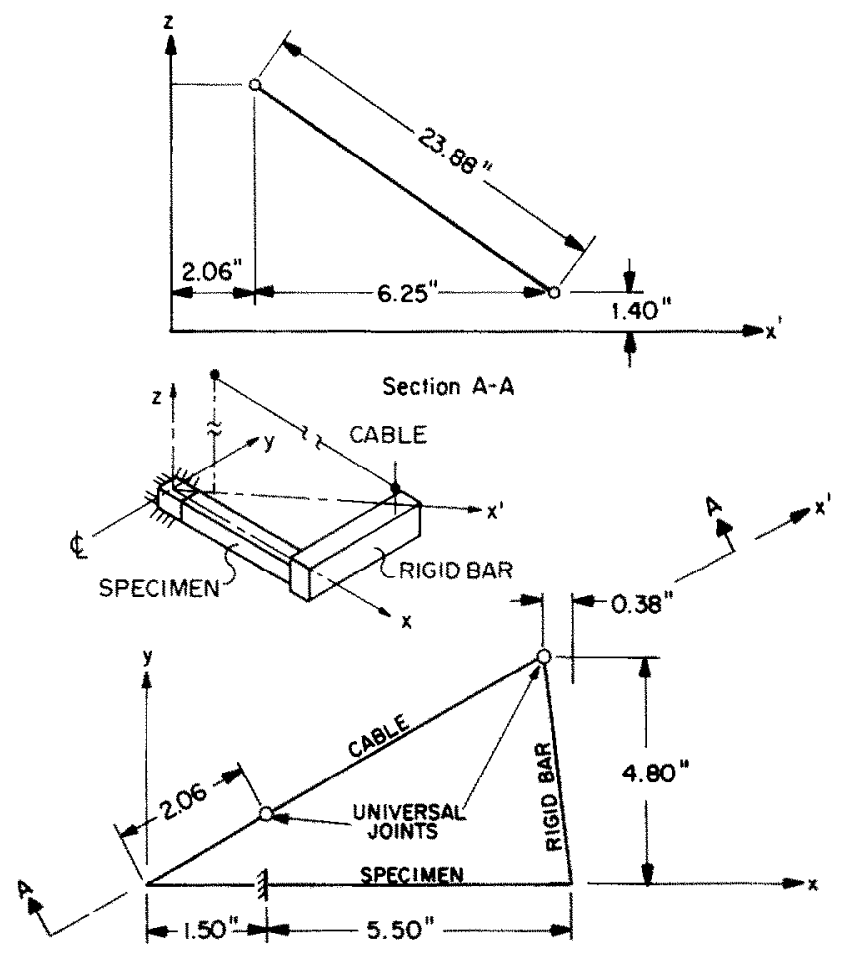

Fig. 9. Initial geometry-combined loading experiment.

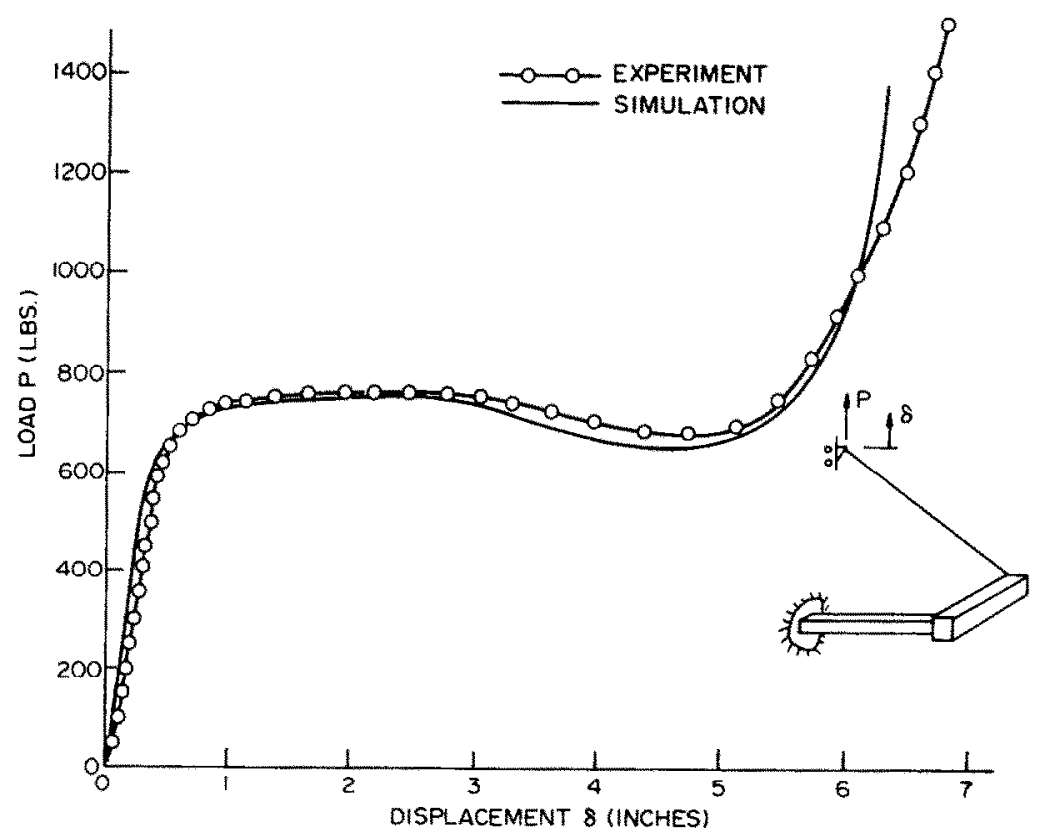

Fig. 10. Computed and experimental force deformation curve. 


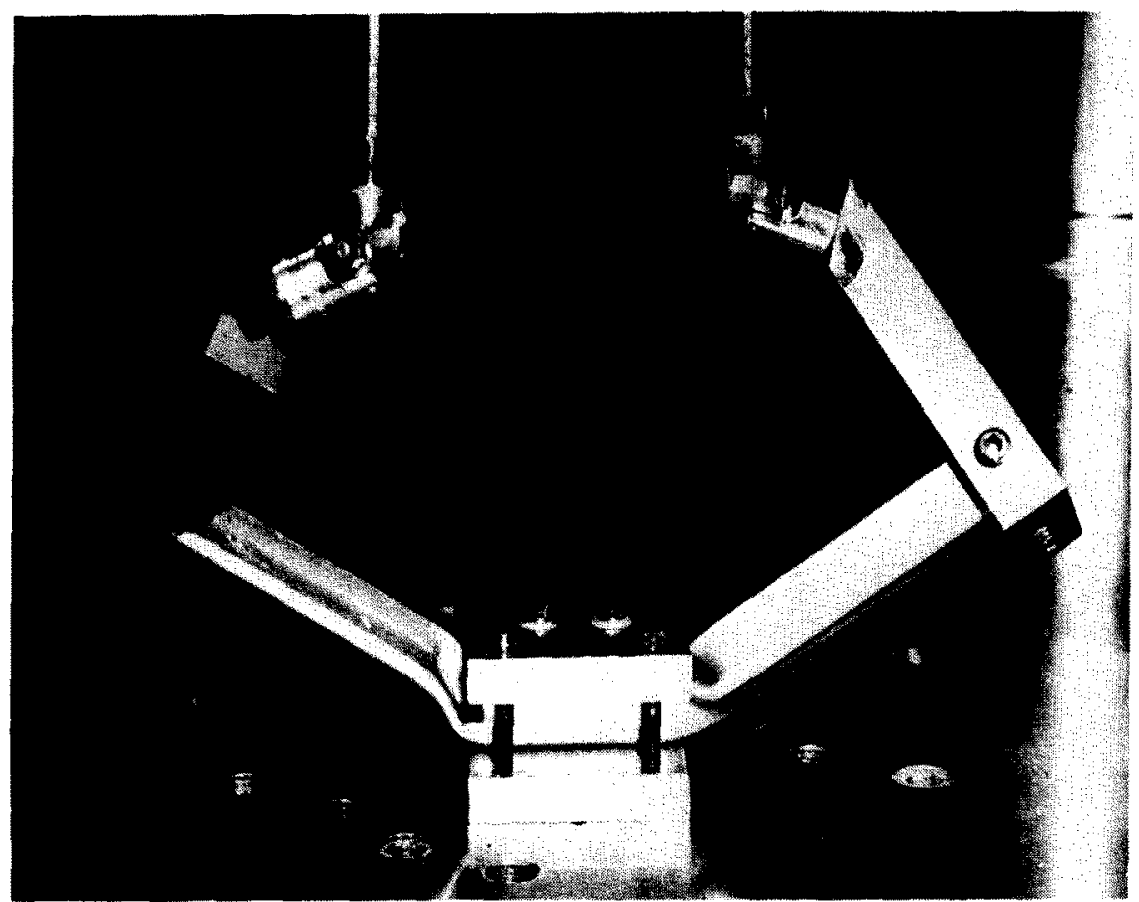

Fig. 11. Final deformed shape-combined loading experiment.

then forms at the root of the cantilever under essentially combined bending and torsion. Torsional rotation dominated early in the hinging action with bending becoming more pronounced with increasing deformation. At very large displacements the load becomes more alighed with the tube axis accounting for the rapid hardening of the system for displacements greater than five inches. Throughout the test, changes in geometry tend to harden the system. Over most of the test this is counteracted by the decrease in the load carrying capacity of the hinge. The final deformed shape is shown in Fig. 11. The rotation in both bending and torsion exceeded $45^{\circ}$.

The computed force-deformation curve using the theory proposed in [4] is compared with the test result in Fig. 10. Agreement is good over the entire deformation range.

Acknowledgment-This work was performed under contract with the National Highway Traffic Safety Administration, U.S. Department of Transportation, Contract No. DOT-HS-4-00855.

\section{REFERENCES}

1. W. Prager, An Introduction to Plasticity. Addison-Wesley, Reading, Mass. (1959).

2. P. G. Hodge, Plastic Analysis of Structures. McGraw-Hill, New York (1959).

3. A. Mendelson, Plasticity Theory and Application, Macmillan, New York (1968).

4. I. K. McIvor, A. S. Wineman and H. C. Wang, P lastic collapse of general frames. Int. J. Solids and Structures. To be published. 